\title{
Temperature Driven Transformation in Dextran-Graft- PNIPAM/Embedded Silver Nanoparticle Hybrid System
}

\author{
V. Chumachenko, ${ }^{1}$ N. Kutsevol ${ }^{D},{ }^{1}$ Iu Harahuts, ${ }^{1}$ D. Soloviov, ${ }^{2}$ L. Bulavin, ${ }^{2}$ O. Yeshchenko, ${ }^{2}$ \\ A. Naumenko $\left(\mathbb{D},{ }^{2}\right.$ O. Nadtoka $(1),{ }^{1}$ and A. Marinin ${ }^{3}$ \\ ${ }^{1}$ Faculty of Chemistry, Taras Shevchenko National University of Kyiv, 01601 64/13 Volodymyrska St., Kyiv, Ukraine \\ ${ }^{2}$ Faculty of Physics, Taras Shevchenko National University of Kyiv, 01601 64/13 Volodymyrska St., Kyiv, Ukraine \\ ${ }^{3}$ Problem Research Laboratory, National University of Food Technology, Volodymyrska Str. 68, 01601 Kyiv, Ukraine
}

Correspondence should be addressed to A. Naumenko; a_naumenko@univ.kiev.ua

Received 7 February 2019; Revised 17 April 2019; Accepted 21 May 2019; Published 12 June 2019

Guest Editor: Marco Caniato

Copyright (c) 2019 V. Chumachenko et al. This is an open access article distributed under the Creative Commons Attribution License, which permits unrestricted use, distribution, and reproduction in any medium, provided the original work is properly cited.

\begin{abstract}
During the last decade, stimuli-responsible polymers based on poly( $\mathrm{N}$-isopropylacrylamide) having conformational transition in the range of physiological temperature have been discussed as novel drug delivery nanosystems. A star-like copolymer with a dextran core and grafted poly(N-isopropylacrylamide) arms (D-g-PNIPAM) was synthesized, characterized, and used as a matrix for silver sol preparation. The comparative study of the behavior of individual D-g-PNIPAM and the nanohybrid system D-g-PNIPAM/silver nanoparticles has been done in the temperature range near the lower critical solution temperature (LCST). The methods of Dynamic Light Scattering, small angle X-ray scattering, and UV-VIS absorption spectroscopy have been used. The existence of single nanoparticles and aggregated nanoparticles located in a limited polymer macromolecular volume was established. The increase of the temperature leads to slight aggregation of the silver nanoparticles at the LCST transition. Single nanoparticles do not aggregate with the temperature increase. The thermally induced collapse of end-grafted poly(N-isopropylacrylamide) chains above the LCST do not affect significantly the size characteristics of silver nanoparticles incorporated into the polymer matrix.
\end{abstract}

\section{Introduction}

The growing progress in nanotechnology and the life sciences demonstrates an urgent need for novel advanced hybrid materials composed of biocompatible polymers and inorganic components [1-3]. The development of nanometersized materials that can perform a desired action upon a local or external stimulus is one major goal of bionanotechnology [4]. Temperature-sensitive polymers allow the creation of locally controlled actuators that can have various applications [4-7].

In recent years, the poly( $\mathrm{N}$-isopropylacrylamide $)$ (PNIPAM) polymer became a subject of study as a promising base for fabrication of nanocomposites for biomedical application [8-10]. Linear PNIPAM is a thermoresponsive polymer widely known for its lower critical solution temperature
(LCST) phenomenon at $32^{\circ} \mathrm{C}$ in aqueous solutions. PNIPAM has coil-to-globule transition at LCST and applies a pore opening and closing mechanism to the porous particles [11]. This mechanism helps in the temperature-triggered release of the loaded molecules into a polymer matrix. The transition temperature for linear PNIPAM is very close to the human skin; thus, this polymer can be applied for photodynamic anticancer therapy $[12,13]$. For a larger window of applications, especially in the field of nanotechnology as drug delivery systems, LCST should be shifted to higher temperatures. It was shown that the branched structure of PNIPAM-containing polymers opened the possibility to tune the LCST $[14,15]$. Also, it was established that the star-like copolymer dextrangraft-PNIPAM can be used as a universal platform for drug delivery, namely, dextran-PNIPAM+doxorubicin nanoparticles have huge potential as novel anticancer agents [16]. 
Hybrid nanocomposites containing polymer and noble metal nanoparticles attract a great interest as material with unique properties [17-20]. It is known that Ag nanoparticles (AgNPs) possess a remarkable biomedical application [20]. The hybrid materials with Ag nanoparticles incorporated into a thermosensitive polymer matrix could be a real achievement in drug delivery. They can combine the local chemotherapy and antibacterial therapy with photothermal anticancer treatment. That can lead to shortening the treatment time and decreasing the drug dosage.

However, successful applications of such intelligent polymers-nanocarriers depend on the possibility to control the hydrophobic-hydrophilic balance of the macromolecule at the physiological temperature. Earlier, the possible tuning of the region of phase transition and the size of hydrophobic domains by variation of the star-like copolymer molecular structure was reported [21]. Also, it was shown that star-like copolymers are more efficient for stable nanosystem preparation in comparison with their linear analogue $[22,23]$.

In the present study, we discuss a behavior of the nanosystem consisting of Ag nanoparticles embedded into a branched PNIPAM matrix in the region of conformational transition of the polymer.

\section{Experimental}

2.1. Polymer Matrix Dextran-Graft-PNIPAM (D-g-PNIPAM). The star-like copolymer consisted of dextran core with $\mathrm{Mw}=70 \times 10^{4} \mathrm{~g} / \mathrm{mol}$, and 15 PNIPAM grafts were used for the AgNP preparation. Further, this copolymer will be labeled as D-g-PNIPAM. Synthesis and characterization of dextran-graft-poly( $\mathrm{N}$-isopropylacrylamide) copolymers were reported in [21].

2.2. D-g-PNIPAM/Ag Nanosystem Synthesis. Reduction of Ag ions was performed in aqueous solutions of the polymer template D-g-PNIPAM. The molar ratio of acrylamide mers to $\mathrm{Ag}$ ions was equal to 5. $0.01 \mathrm{M} \mathrm{AgNO} 3$ aqueous solution was added to $0.5 \mathrm{ml}$ of polymer solution $\left(C=1 \mathrm{gl}^{-1}\right)$ and stirred for $20 \mathrm{~min}$ at $T=25^{\circ} \mathrm{C}$. Then, $0.1 \mathrm{M}$ of $\mathrm{NaBH} 4$ was added drop by drop while stirring. The solution turned reddish brown, which indicated the formation of AgNPs. The obtained Ag sols were stored in cold dark.

2.3. Transmission Electron Microscopy (TEM). For the sample preparation, 400 mesh $\mathrm{Cu}$ grids with plain carbon film were rendered hydrophilic by a glow discharge treatment (ELMO, Cordouan Technologies, Bordeaux, France). A $5 \mu$ l drop was deposited and allowed to be adsorbed for $1 \mathrm{~min}$, then the excess of the solution was removed with a piece of filter paper. The observations of the AgNPs were carried on two TEMs, Tecnai G2 or CM12 (FEI, Eindhoven, Netherlands), and the images were acquired with a ssCCD Eagle camera on the Tecnai and a MegaView SIS Camera on the CM12.

2.4. Dynamic Light Scattering (DLS). DLS measurements were carried out by using a Zetasizer Nano ZS90 (Malvern Instruments Ltd., UK). The apparatus contains a $4 \mathrm{~mW}$ $\mathrm{He}-\mathrm{Ne}$ laser with a wavelength $632.8 \mathrm{~nm}$, and the scattered light is detected at an angle $173^{\circ}$ (back scattering).
For accurate study of transition, correlograms of $0.1 \mathrm{mg} / \mathrm{ml}$ aqueous D70-PNIPAM were collected in the temperature range of $25-40^{\circ} \mathrm{C}$ with a step of $0.1^{\circ} \mathrm{C}$ and heat rate of $0.02^{\circ} \mathrm{C} / \mathrm{min}$. Each temperature point was held for $5 \mathrm{~min}$ before measurements to equilibrate the sample. 10 correlation curves for each temperature point were treated by the CONTIN algorithm [24] which is known to be reliable in getting the hydrodynamic diameter (DH) distributions for complicated systems [24]. The CONTIN algorithm was performed using the MATLAB program rilt.m [25].

2.5. Small Angle X-Ray Scattering (SAXS). The investigations by the small angle X-ray (SAXS) method were carried out on a Rigaku instrument which is available at the Moscow Institute of Physics and Technology (Dolgoprudny, Russia) [26].

2.6. UV-VIS Spectroscopy. UV-VIS absorption spectra of AgNPs in the D70-g-PNIPAM/AgNP nanosystem were recorded by the Cary 60 UV-VIS spectrophotometer (Agilent Technologies Inc.) using 1:25 dilutions in the temperature range of $23-45^{\circ} \mathrm{C}$. The measurements were performed with the temperature step of $1^{\circ} \mathrm{C}$ with the temperature stabilization in each temperature point during $5 \mathrm{~min}$.

\section{Results and Discussion}

Earlier it was reported [21] that the star-like structure of PNIPAM-containing copolymers allows to shift LCST to a higher temperature, namely, to 33.7 instead of $32^{\circ} \mathrm{C}$ for linear PNIPAM. The D-g-PNIPAM copolymer with dextran core $\left(\mathrm{Mv}=7 \times 10^{5} \mathrm{~g} / \mathrm{mol}\right)$ and 15 PNIPAM grafts were chosen for synthesis of a hybrid nanosystem with further precise analysis of temperature-induced changes in the polymer/AgNP nanosystem, since this polymer revealed well-defined size changes in the range of $25-40^{\circ} \mathrm{C}$ [21]. Molecular parameters of this copolymer, determined by SEC, are as follows: $\mathrm{Mv}=1.05 \times 10^{-6} \mathrm{~g} / \mathrm{mol}, \mathrm{Mn}=0.674 \times 10^{-6} \mathrm{~g} / \mathrm{mol}$, and $\mathrm{Mw} / \mathrm{Mn}=1.52$.

The TEM image of the synthesized Ag sol into the solution of the D-g-PNIPAM copolymer is represented in Figure 1. It is seen that there are single nanoparticles and nanoparticles located in the limited volume. Obviously, these nanoparticles are located inside of the macromolecules. It should be noted that the synthesis of Ag nanoparticles was performed in dilute polymer solutions, thus, below the overlap concentration.

DLS was efficiently used for the accurate study of the conformational transition process for individual D-g-PNIPAM in water solution [21] and for the study of Au sols synthesized in the solution of the D-g-PNIPAM copolymer [27]. Therefore, this method has been chosen for the characterization of the nanosystem D-g-PNIPAM/AgNPs below and above the LCST of the polymer matrix.

The DLS experiment was carried out in the temperature range $25-40^{\circ} \mathrm{C}$ with a step of $0.1^{\circ} \mathrm{C}$ and a heating rate of $0.02^{\circ} \mathrm{C} / \mathrm{min}$. For the nanosystem D-g-PNIPAM/AgNPs, the size distribution curves exhibit a more complicated character than for those of the individual D-g-PNIPAM in water solution [21]. DLS results for D-g-PNIPAM/AgNP 


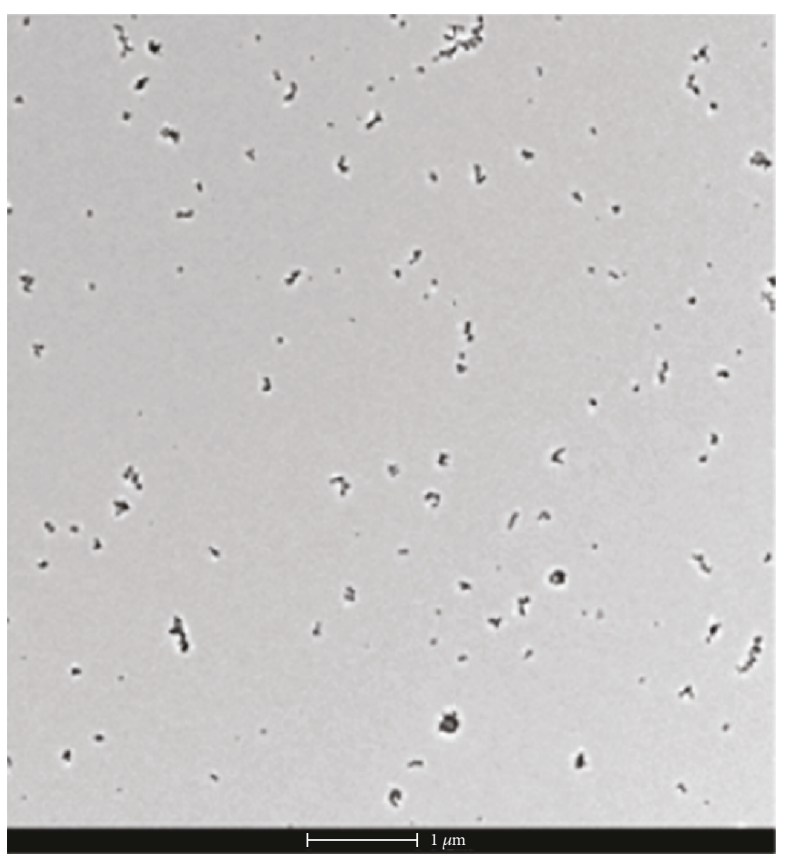

(a)

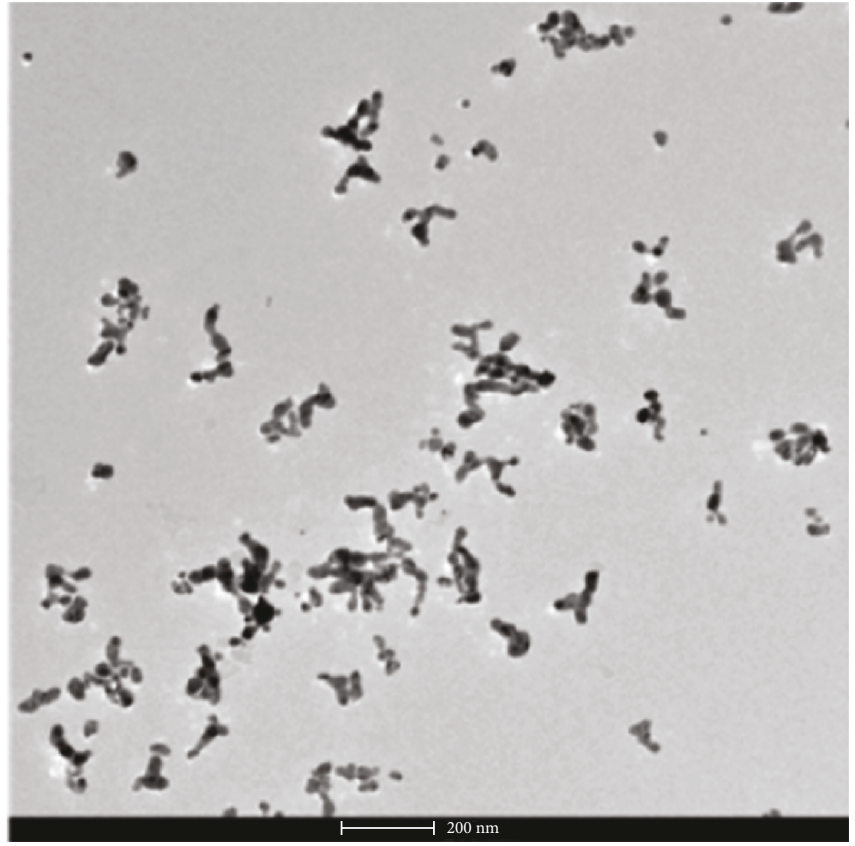

(b)

Figure 1: TEM image of Ag sol, synthesized in D-g-PNIPAM solution.

nanosystems revealed the presence of few types of scattering nanoobjects. The hydrodynamic diameter distributions for D-g-PNIPAM/AgNP nanosystems at $25^{\circ} \mathrm{C}$ are shown in Figure 2. The first peak in the size distribution scattering curve corresponds to the individual AgNPs; further, they will be indicated as Nanoobjects 1 . The sizes of Nanoobjects 1 are estimated as $5-10 \mathrm{~nm}$. The second peak corresponds to Nanoobjects 2. Nanoobjects 2 are $85-100 \mathrm{~nm}$ in size at $25^{\circ} \mathrm{C}$ and correspond to the aggregates of D-g-PNIPAM macromolecules with incorporated AgNPs inside. It is evident that the size of individual macromolecules of D-g-PNIPAM is equal to $37-40 \mathrm{~nm}[21]$ at this temperature.

The Peak 1 of individual AgNPs was registered for all studied temperature ranges and revealed the presence of nanoparticles of 5-10 nm in size (Figure 2; black, red, and green curves). The drastic change in the hydrodynamic diameter of Nanoobjects 2 (Peak 2) and the variation in intensity of Peak 1 and Peak 2 were observed during the heating process (from 25 to $40^{\circ} \mathrm{C}$ ). It is reasonable to take into account the peak positions, since their intensities depend on the density of the scattering object. This parameter for hybrid nanosystem D-g-PNIPAM/AgNPs depends on two factors: the conformation of the polymer matrix and the distance between incorporated AgNPs. Both these parameters are changed in the region of LCST.

At the temperature of $32^{\circ} \mathrm{C}$, we observe the drastic decrease of the size of Nanoobjects 2 up to $40 \mathrm{~nm}$. Such diminished size of the scattering objects is caused by conformational transition of the polymer matrix (partial collapse of the macromolecule). Further heating to $40^{\circ} \mathrm{C}$ leads to the increase in size of Nanoobjects 2 to $200 \mathrm{~nm}$ and increases their polydispersity. Also, the third peak (green curve) in size distribution appears, which may correspond to the slight deviation from sphere-shaped scattering objects. Obviously, we observe the aggregation process in the system, because above LCST, the hydrophilic-hydrophobic balance of the polymer matrix changes. This peak also was observed for individual D-g-PNIPAM [21].

Further, we analyze the Nanoobject 2 (D-g-PNIPAM with incorporated AgNPs) in the studied temperature range, since the change in size of Nanoobject 1 (free AgNPs) was not observed in the studied temperature range. Figure 3 demonstrates the comparative behavior of individual D-g-PNIPAM macromolecules and Nanoobjects 2 (D-gPNIPAM/AgNPs) within the temperature range $25-40^{\circ} \mathrm{C}$. It is seen that the shape of both curves is similar. LCST for both systems is registered at $33.8^{\circ} \mathrm{C}$ for both systems.

Thus, the presence of silver nanoparticles into the D-g-PNIPAM matrix leads to the formation of nanoobjects consisting of few macromolecules. It can be assumed that the presence of AgNPs inside the polymer can block some hydrophilic groups causing a partial decrease of the hydrophilicity of the D-g-PNIPAM matrix and formation of aggregates. There are two types of AgNPs: free (single) nanoparticles formed outside of macromolecules, and nanoparticles incorporated inside of the polymer macromolecule. It was shown that free nanoparticles do not aggregate with temperature increase. However, the DLS data analysis does not give a reply to the main question. Does collapse of macromolecules cause some aggregation process of the incorporated AgNPs or does it only lead to a change in the distance between them?

Further, SAXS and UV-Vis spectroscopy were used for analysis of the behavior of D-g-PNIPAM/AgNP nanosystems in the region of LCST. Figure 4 shows SAXS curves measured on a Rigaku instrument at different temperatures 


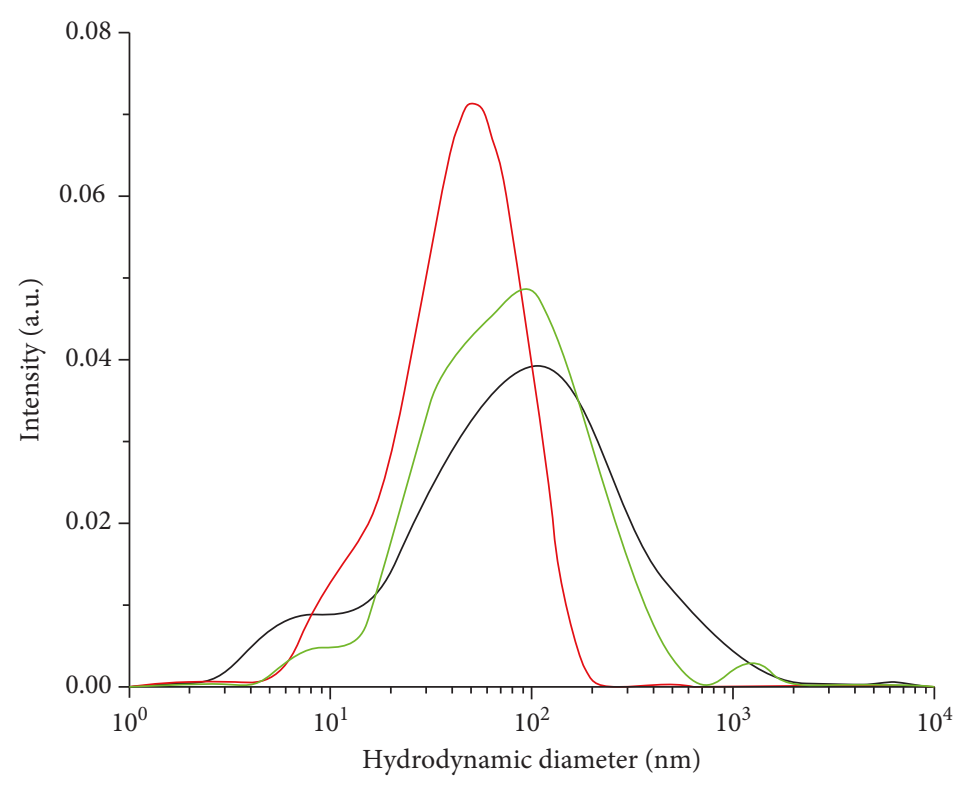

Figure 2: Hydrodynamic diameter distributions for D-g-PNIPAM/AgNP nanosystems at $25^{\circ} \mathrm{C}$ (black curve), $32^{\circ} \mathrm{C}$ (red curve), and $40^{\circ} \mathrm{C}$ (green curve).

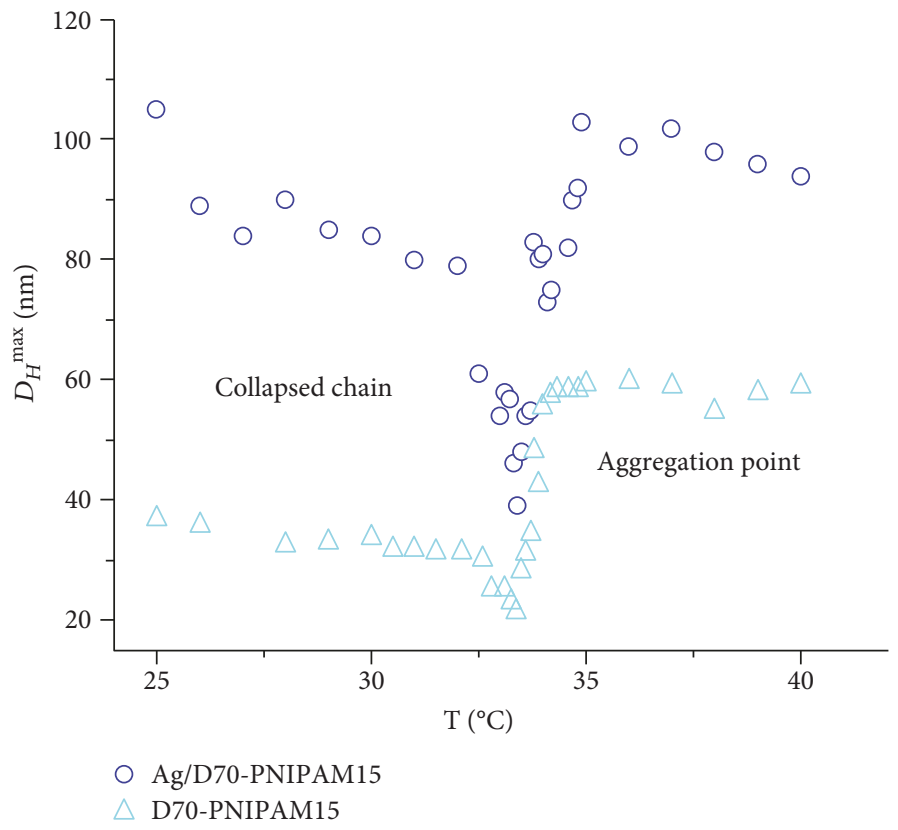

FIGURE 3: Temperature dependence of the hydrodynamic diameter for D-g-PNIPAM (individual macromolecule, black empty squares) and D-g-PNIPAM/AgNP nanosystems (Nanoobject 2, blue empty circles).

and on a BioSAXS instrument (at ESRF) at $25^{\circ} \mathrm{C}$. One can see that curves from different instruments at $25^{\circ} \mathrm{C}$ are similar. So $\mathrm{P}(\mathrm{R})$ functions for these curves should also be similar. Anyway, the main goal of our SAXS measurements was investigation of a sample structure at different temperatures. Since SAXS curves do not change with temperature change, the sample structure is also stable. Curves were normalized on transmission; the buffer (D-g-PNIPAM solution with the same concentration and without AgNPs) was subtracted. It should be noted that the used concentra- tion of the polymer in the solution is invisible for SAXS (the scattering curve is similar to the scattering curve of distilled water). That is why all curves in Figure 4 correspond to the scattering on AgNPs. One can see that temperature changes of scattering curves are minor. It means that the structure of the AgNPs, their distribution, and interactions (including aggregation) do not change in this temperature range. Obviously, the diminished distance between AgNPs in the collapsed polymer matrix above LCST takes place. 


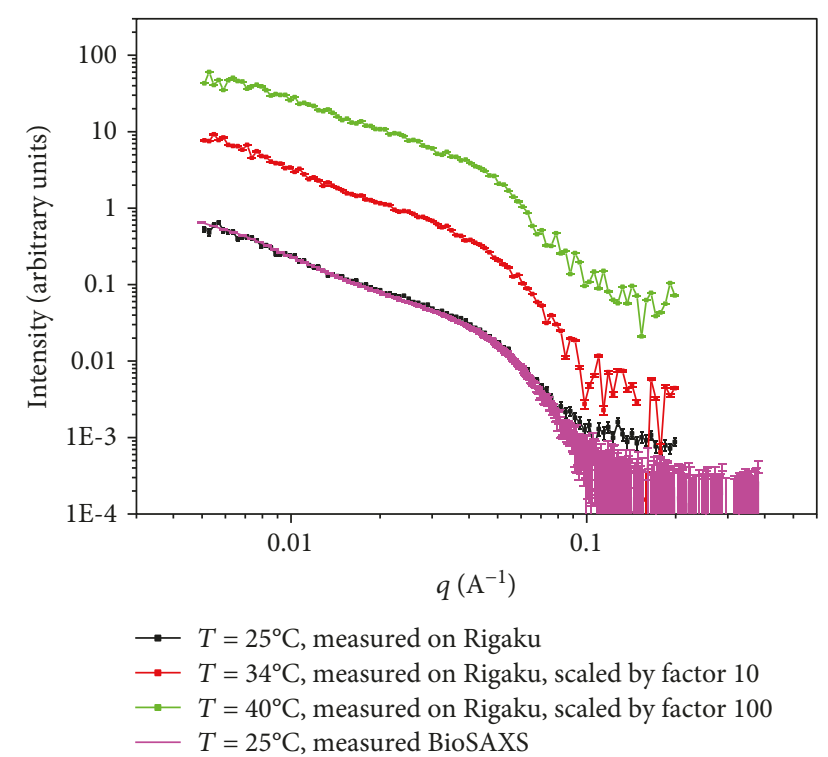

FIGURE 4: SAXS curves, measured at different temperatures on a Rigaku instrument: black: $25^{\circ} \mathrm{C}$, red: $34^{\circ} \mathrm{C}$, and green: $40^{\circ} \mathrm{C}$. Magenta: SAXS curve, measured with the BioSAXS instrument at $\mathrm{ESRF}$ at $25^{\circ} \mathrm{C}$ (added for comparison).

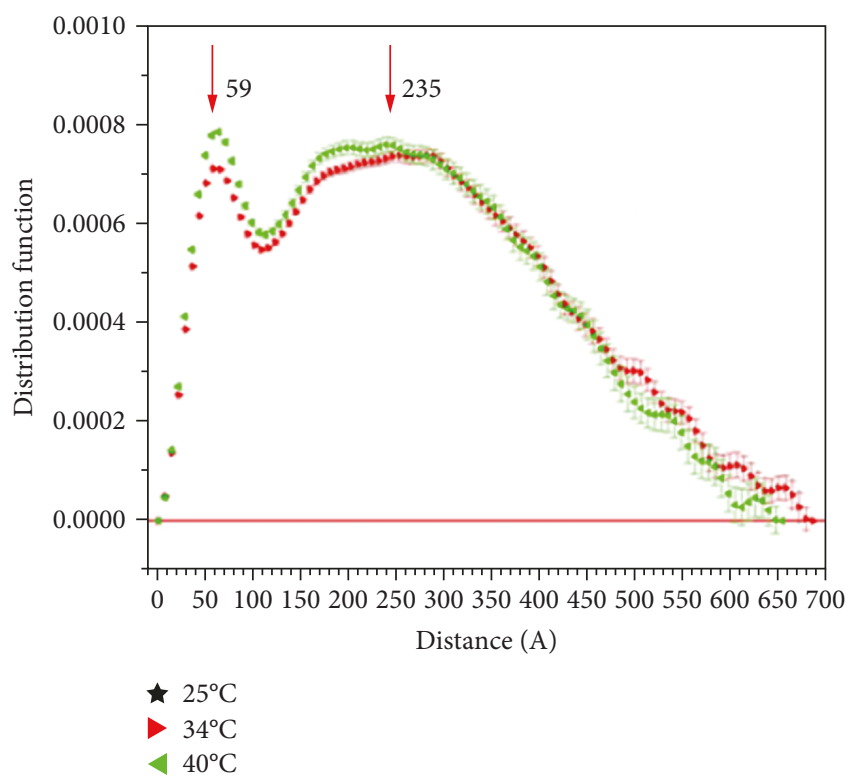

FIgURE 5: Distance distribution functions of D-g-PNIPAM/AgNP nanosystems at different temperatures: $25^{\circ} \mathrm{C}$ (black curve), $32^{\circ} \mathrm{C}$ (red curve), and $40^{\circ} \mathrm{C}$ (green curve).

Using the PRIMUS program from the ATSAS package $[28,29]$, we calculated the distance distribution functions (DDFs) for each temperature (Figure 5). All DDFs have two peaks-at $5.9 \mathrm{~nm}$ and $23.5 \mathrm{~nm}$. They correspond to two main particle sizes in our solution. DDFs of AgNPs at $25^{\circ} \mathrm{C}$ and $34^{\circ} \mathrm{C}$ are almost the same and differ a little at $40^{\circ} \mathrm{C}$. Anyway, these changes are negligibly small.
TABLE 1: Comparison of particle sizes, obtained from Dynamic Light Scattering data and distance distribution function calculations.

\begin{tabular}{lcc}
\hline & Size 1 & Size 2 \\
\hline DLS & $5-10 \mathrm{~nm}$ & $80-100 \mathrm{~nm}$ \\
& (in full temperature range) & (in full temperature range) \\
DDF & $5.9 \mathrm{~nm} \pm 20 \%$ & $23.5 \mathrm{~nm} \pm 10 \%$ \\
\hline
\end{tabular}

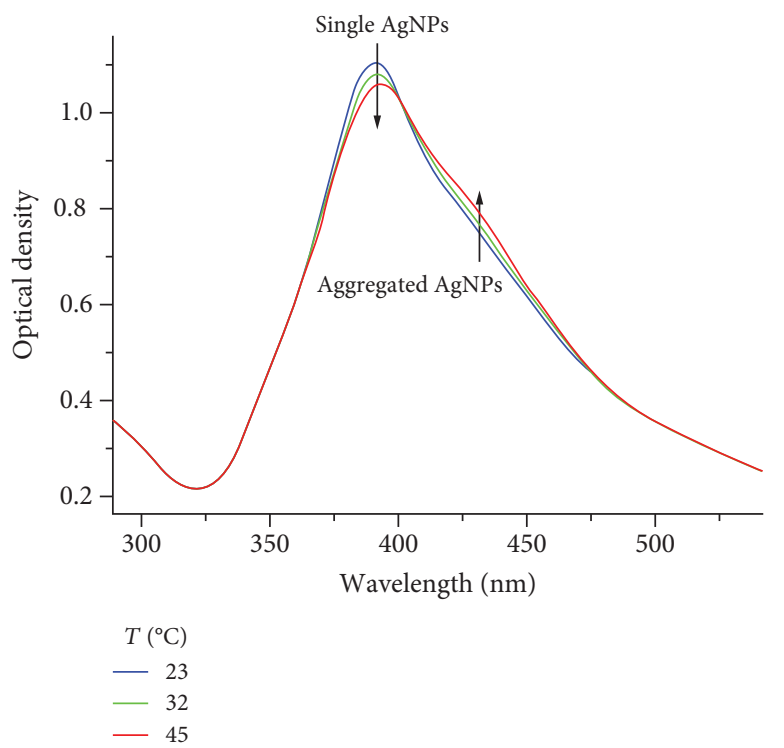

FIgURE 6: The behavior of the SPR absorption peak of AgNPs in the D70-g-PNIPAM/AgNP nanosystem at the increase of the temperature from $23^{\circ} \mathrm{C}$ to $45^{\circ} \mathrm{C}$.

Comparing particle sizes obtained from DDF (Figure 5) with sizes obtained from DLS measurements, one can say that the Size 1 (Table 1) from both methods is in good agreement. Thus, it may indicate that Size 1 (corresponding to Peak 1, Figure 2) is the size of free AgNPs. At the same time, DLS and DDF give a completely different Size 2 each. As we already mentioned, the DLS method is sensitive both to polymer size and to AgNP sizes and SAXS is sensitive only to AgNP sizes. Considering this, we can conclude that the Size 2 obtained from the DLS data (Peak 2, Figure 1) corresponds to the polymer matrix dimension (which changes with temperature) and Size 2 obtained from DDF calculations corresponds to the size of AgNPs incorporated in the polymer matrix. These AgNPs are located in local volume of the macromolecule.

To study the transformations occurring at the conformational phase transition in the D70-g-PNIPAM/AgNP nanosystem, the surface plasmon resonance (SPR) in AgNPs was used as an optical sensor of these transformations. Namely, the temperature-caused behavior of the SPR absorption peak was analyzed in the temperature range $23^{\circ} \mathrm{C}$ to $45^{\circ} \mathrm{C}$. The temperature behavior of the AgNP absorption spectrum under the increasing temperature is shown in Figure 6. It is seen that the SPR spectrum has a two-component structure. 
The high-energy one with a maximum at $390 \mathrm{~nm}$ (at room temperature of $23^{\circ} \mathrm{C}$ ) corresponds to single AgNPs. The low-energy SPR peak with a maximum at $435 \mathrm{~nm}$ (at $23^{\circ} \mathrm{C}$ ) corresponds to the light absorption by the aggregates of AgNPs. Meanwhile, the quite large fraction of AgNPs in a hybrid system exists in the form of the aggregates that caused an appearance of additional low-energy peak corresponding to aggregated AgNPs. Such two-component structure of the plasmonic absorption spectrum in the D70-g-PNIPAM/AgNP nanosystem is similar to the one observed in our recent work [30] where the laser-driven phase transformations were studied in D70-g-PNIPAM/AuNPs containing AuNPs.

It is seen from Figure 6 that similar to the data of the SAXS measurements shown in Figure 5, the changes in the plasmonic absorption spectrum of AgNPs are quite small. However, let us note that the SPR absorption spectrum changes during the heating of the sample. Namely, the intensity of the high-energy peak corresponding to single NPs decreases at the increase of the temperature. Meanwhile, the intensity of the low-energy peak corresponding to aggregated AgNPs increases at the increase of the temperature. Similar behavior was observed for the plasmonic absorption spectrum in D70-g-PNIPAM/AuNPs reported in our recent work [30]. Similar to the results of reference [30], the observed transformation of the plasmonic absorption spectrum proves the fact of the aggregation of AgNPs occurring during the conformational LCST phase transition. Indeed, the shrinking of D70-g-PNIPAM macromolecules leads to decrease of the distance between the Ag NPs and their consequent aggregation. The fraction of single AgNPs decreases causing the decrease of the high-energy peak, while the fraction of the aggregated AgNPs increases causing the increase of the low-energy peak intensity. Thus, one can conclude that SPR spectroscopy proves the fact of enforcing the process of the AgNP aggregation during the phase transition in D70-g-PNIPAM/AgNP nanosystems.

\section{Conclusions}

The star-like copolymer dextran-graft-poly(N-isopropylacrylamide) has been used as a matrix for D-g-PNIPAM/AgNP nanosystem fabrication. It was shown that the branched structure of host PNIPAM polymers allowed tuning of LCST to higher values of $T=33.8^{\circ} \mathrm{C}$ in comparison with linear PNIPAM. The existence of two types of AgNPs into the D-g-PNIPAM/AgNP nanosystem was revealed: single (separated) nanoparticles and aggregated nanoparticles. The comparative study of individual D-g-PNIPAM and D-g-PNIPAM/Ag nanosystems in water medium showed that Ag nanoparticles incorporated into the D-g-PNIPAM matrix did not affect the range of LCST. It was shown that slight aggregation of $\mathrm{Ag}$ nanoparticles occurs due to the macromolecule shrinking at LCST. It was obtained that the thermally induced collapse of end-grafted poly $(\mathrm{N}$-isopropylacrylamide) chains above the LCST did not affect significantly the size characteristics of silver nanoparticles incorporated into the polymer matrix. It can be concluded that such nanosystems can be a promising approach for the preparation of nanocomposites containing nanoparticles which can be used as a nanocarrier for biomedical application.

\section{Data Availability}

(1) The data concerning the synthesis and characterization of the polymer matrix have been published in Journal of Molecular Liquids (2017) and cited in the text as reference [21]. (2) Some of the new data are under commercialization, and the others are the part of $\mathrm{PhD}$ thesis (auth. Iu Harahuts) which is under preparation. (3) If anybody needs the data, it can be asked from the corresponding author.

\section{Conflicts of Interest}

The authors declare that there is no conflict of interest regarding the publication of this paper.

\section{Acknowledgments}

The authors are grateful to Legros Mélanie from the Institut Charles Sadron (Strasbourg, France) for the branched PNIPAM sample characterization by Size Exclusion Chromatography. The authors are grateful to A. Kuklin from the Moscow Institute of Physics and Technology (Dolgoprudny, Russia) for sample characterization by the small angle X-ray (SAXS) method. This publication is supported in part by the Grant of the State Fund for Fundamental Research (Ukraine), project $\Phi 76 / 64-2017$ "New Multifunctional Hybrid Nanocomposites for Photodynamic Chemotherapy of Tumor Cells." O.A. Yeshchenko confirms the support by NATO Science for Peace and Security (SPS) Program (grant NUKR.SFPP 984617).

\section{References}

[1] A. P. Nikalje, "Nanotechnology and its applications in medicine," Medicinal Chemistry, vol. 5, no. 2, pp. 081-089, 2015.

[2] A. Beriso, "Early cancer detection and treatment with nanotechnology," Journal of Nanomaterials \& Molecular Nanotechnology, vol. 6, no. 5, 2017.

[3] S. Bhatia, "Nanoparticles types, classification, characterization, fabrication methods and drug delivery applications," in Natural Polymer Drug Delivery Systems, pp. 33-93, Springer International Publishing, Cham, 2016.

[4] M. R. Aguilar, C. Elvira, A. Gallardo, B. Vázquez, and J. S. Román, "Smart polymers and their applications as biomaterials. III Biomaterials," in Topics in Tissue Engineering, N. Ashammakhi, R. Reis, and E. Chiellini, Eds., vol. 3, pp. 1-27, University of Oulu, Finland, 2007, Chapter 6.

[5] A. Gandhi, A. Paul, S. O. Sen, and K. K. Sen, "Studies on thermoresponsive polymers: phase behaviour, drug delivery and biomedical applications," Asian Journal of Pharmaceutical Sciences, vol. 10, no. 2, pp. 99-107, 2015.

[6] M. A. Ward and T. K. Georgiou, "Thermoresponsive polymers for biomedical applications," Polymers, vol. 3, no. 3, pp. 12151242, 2011.

[7] O. Sedláček, P. Černoch, J. Kučka et al., "Thermoresponsive polymers for nuclear medicine: which polymer is the best?," Langmuir, vol. 32, no. 24, pp. 6115-6122, 2016. 
[8] Y. M. Ma, D. X. Wei, H. Yao, L. P. Wu, and G. Q. Chen, "Synthesis, characterization and application of thermoresponsive polyhydroxyalkanoate-graft-poly(N-isopropylacrylamide)," Biomacromolecules, vol. 17, no. 8, pp. 2680-2690, 2016.

[9] K. N. Plunkett, X. Zhu, J. S. Moore, and D. E. Leckband, "PNIPAM chain collapse depends on the molecular weight and grafting density," Langmuir, vol. 22, no. 9, pp. 4259-4266, 2006.

[10] A. Galperin, T. J. Long, and B. D. Ratner, "Degradable, thermo-sensitive poly( $\mathrm{N}$-isopropyl acrylamide)-based scaffolds with controlled porosity for tissue engineering applications," Biomacromolecules, vol. 11, no. 10, pp. 2583-2592, 2010.

[11] H. Du, R. Wickramasinghe, and X. Qian, "Effects of salt on the lower critical solution temperature of poly (N-Isopropylacrylamide)," The Journal of Physical Chemistry. B, vol. 114, no. 49, pp. 16594-16604, 2010.

[12] G. Graziano, "On the temperature-induced coil to globule transition of poly- $\mathrm{N}$-isopropylacrylamide in dilute aqueous solutions," International Journal of Biological Macromolecules, vol. 27, no. 1, pp. 89-97, 2000.

[13] V. C. Lopez, J. Hadgraft, and M. J. Snowden, "The use of colloidal microgels as a (trans)dermal drug delivery system," International Journal of Pharmaceutics, vol. 292, no. 1-2, pp. 137-147, 2005.

[14] A. Khanal, M.-P. N. Bui, and S. S. Seo, "Microgel-encapsulated methylene blue for the treatment of breast cancer cells by photodynamic therapy," Journal of Breast Cancer, vol. 17, no. 1, pp. 18-24, 2014.

[15] W. Tao and L. Yan, "Thermogelling of highly branched poly(N-isopropylacrylamide)," Journal of Applied Polymer Science, vol. 118, no. 6, pp. 3391-3399, 2010.

[16] T. Matvienko, V. Sokolova, S. Prylutska et al., "In vitro study of the anticancer activity of various doxorubicin-containing dispersions," BioImpacts: BI, vol. 9, no. 1, pp. 57-63, 2019.

[17] N. Uehara, "Polymer-functionalized gold nanoparticles as versatile sensing materials," Analytical Sciences, vol. 26, no. 12, pp. 1219-1228, 2010.

[18] O. A. Yeshchenko, A. P. Naumenko, N. V. Kutsevol et al., "Anomalous inverse hysteresis of phase transition in thermosensitive dextran-graft-PNIPAM copolymer/Au nanoparticles hybrid nanosystem," Journal of Physical Chemistry C, vol. 122, no. 14, pp. 8003-8010, 2018.

[19] W. Yu, L.-L. Lou, K. Yu, S. Li, Y. Shi, and S. Liu, "Pt nanoparticles stabilized by thermosensitive polymer as effective and recyclable catalysts for the asymmetric hydrogenation of ethyl pyruvate," RSC Advances, vol. 6, no. 57, pp. 52500-52508, 2016.

[20] L. Mei, Z. Lu, X. Zhang, C. Li, and Y. Jia, "Polymer-ag nanocomposites with enhanced antimicrobial activity against bacterial infection," ACS Applied Materials \& Interfaces, vol. 6, no. 18, pp. 15813-15821, 2014.

[21] V. Chumachenko, N. Kutsevol, Y. Harahuts, M. Rawiso, A. Marinin, and L. Bulavin, "Star-like dextran-graft-PNiPAM copolymers. Effect of internal molecular structure on the phase transition," Journal of Molecular Liquids, vol. 235, pp. 77-82, 2017.

[22] N. V. Kutsevol, V. A. Chumachenko, M. Rawiso, V. F. Shkodich, and O. V. Stoyanov, "Star-like polymers dextranpolyacrylamide: the prospects of application for nanotechnol- ogy," Journal of Structural Chemistry, vol. 56, no. 5, pp. 1016-1023, 2015.

[23] M. Bezuglyi, N. Kutsevol, M. Rawiso, and T. Bezugla, "Watersoluble branched copolymers dextran-polyacrylamide and their anionic derivates as matrices for metal nanoparticles in situ synthesis," Chemik, vol. 66, no. 8, pp. 862-867, 2012.

[24] A. Scotti, W. Liu, J. S. Hyatt et al., "The CONTIN algorithm and its application to determine the size distribution of microgel suspensions," The Journal of Chemical Physics, vol. 142, no. 23, pp. 234905-234913, 2015.

[25] O. Peña-Rodríguez, P. P. González Pérez, and U. Pal, "MieLab: a software tool to perform calculations on the scattering of electromagnetic waves by multilayered spheres," International Journal of Spectroscopy, vol. 2011, Article ID 583743, 10 pages, 2011.

[26] D. V. Soloviov and V. I. Gordeliy, "Low resolution structural studies of apoferritin via SANS and SAXS: the effect of concentration," Journal of Optoelectronics and Advanced Materials, vol. 17, no. 9-10, pp. 1397-1402, 2015.

[27] N. Kutsevol, A. Glamazda, V. Chumachenko et al., "Behavior of hybrid thermosensitive nanosystem dextran-graftPNIPAM/gold nanoparticles: characterization within LCTS," Journal of Nanoparticle Research, vol. 20, no. 9, p. 236, 2018.

[28] L. Bulavin, N. Kutsevol, V. Chumachenko, D. Soloviov, A. Kuklin, and A. Marynin, "SAXS combined with UV-vis spectroscopy and QELS: accurate characterization of silver sols synthesized in polymer matrices," Nanoscale Research Letters, vol. 11, no. 1, p. 35, 2016.

[29] P. V. Konarev, V. V. Volkov, A. V. Sokolova, M. H. J. Koch, and D. I. Svergun, "PRIMUS: a Windows PC-based system for small-angle scattering data analysis," Journal of Applied Crystallography, vol. 36, no. 5, pp. 1277-1282, 2003.

[30] O. A. Yeshchenko, A. P. Naumenko, N. V. Kutsevol, and I. I. Harahuts, "Laser-driven structural transformations in dextran-graft-PNIPAM copolymer/Au nanoparticles hybrid nanosystem: the role of plasmon heating and attractive optical forces," RSC Advances, vol. 8, no. 67, pp. 38400-38409, 2018. 


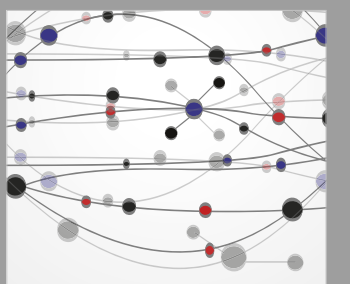

The Scientific World Journal
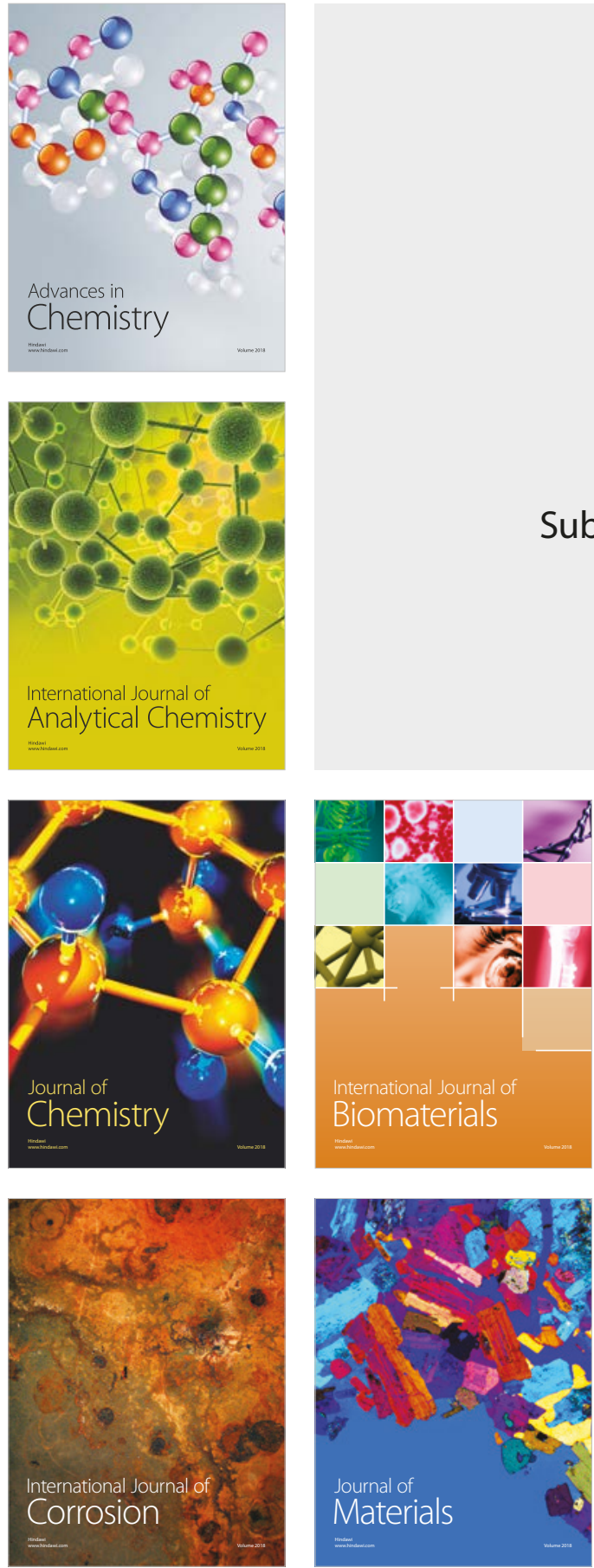

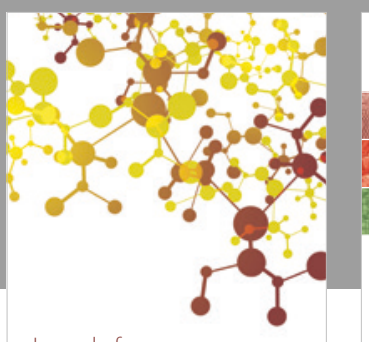

Journal of

Applied Chemistry
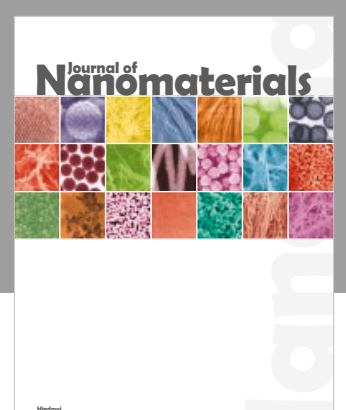

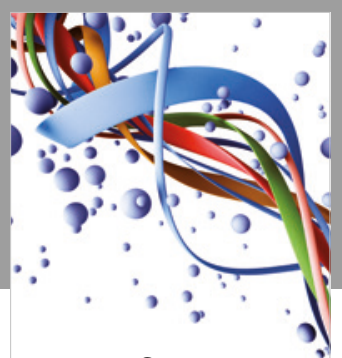

Scientifica

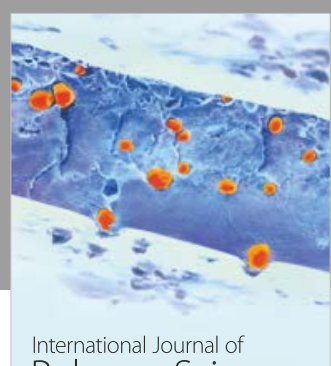

Polymer Science

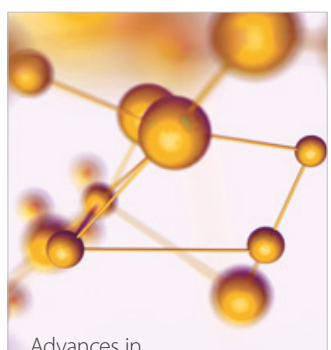

Physical Chemistry
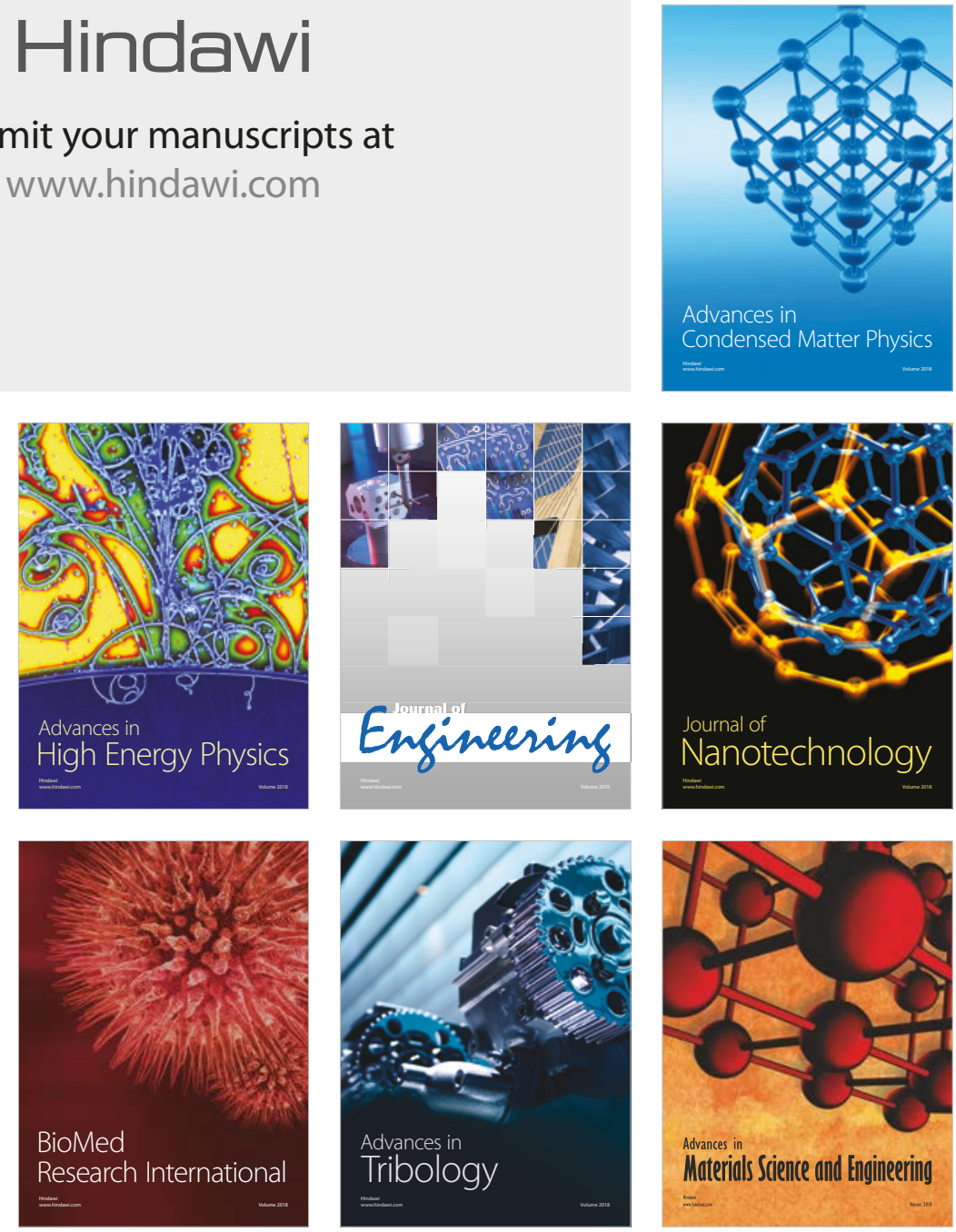